\title{
FACTORIZATION OF MEASURES AND PERFECTION
}

\author{
WOLFGANG ADAMSKI
}

\begin{abstract}
It is proved that a probability measure $P$ defined on a countably generated measurable space $(Y, C)$ is perfect iff every probability measure on $\mathbf{R} \times Y$ having $P$ as marginal can be factored. This result leads to a generalization of a theorem due to Blackwell and Maitra.
\end{abstract}

We characterize countably generated measurable spaces $(Y, C)$ which have the following property: For any measurable space $(X, A)$ and any probability measure $Q$ on the product space $(X \times Y, A \otimes C), Q$ can be factored, $Q=\tilde{Q} \times K$, which means that $\tilde{Q}$ is a probability measure on $(X, A)$ and $K: X \times C \rightarrow[0,1]$ is a transition probability (i.e. $K(\cdot, C)$ is $A$-measurable for every $C \in C$ and $K(x, \cdot)$ is a probability measure on $C$ for every $x \in X)$ such that $Q(A \times C)=\int_{A} K(x, C) \tilde{Q}(d x)$ holds for all $A \in A$ and $C \in C$. For the special case of a separable metric space $Y$ equipped with its Borel $\sigma$-algebra, we obtain the characterization of absolutely measurable separable metric spaces given by Blackwell and Maitra in [1].

In the sequel we shall use the following notation. If $Y$ is a topological space, then $B(Y)$ denotes the Borel $\sigma$-algebra of $Y$. In particular, we denote by $B$ the Borel $\sigma$-algebra of the real line $\mathbf{R}$ with the Euclidean topology. If $(X, A)$ and $(Y, C)$ are measurable spaces, $f: X \rightarrow Y$ is $A, C$-measurable and $\mu$ is a measure on $\mathcal{A}$, then $\mu^{f}$ denotes the image measure of $\mu$ under $f$. A probability measure $P$ defined on a measurable space $(Y, C)$ is said to be perfect if for every $C$-measurable real-valued function $f$ on $Y$, there exists a set $B \in B$ such that $B \subset f(Y)$ and $P\left(f^{-1}(B)\right)=1$. Several other characterizations of perfect measures are given in [5].

We can now prove the main result of this note. Observe that the proof of our implication $(3) \Rightarrow(1)$ is a modification of the proof of the implication (c) $\Rightarrow(a)$ in [1].

THEOREM. Let $(Y, C, P)$ be a probability space. Then the following three statements are equivalent:

(1) $P$ is perfect.

(2) For any measurable space $(X, \mathcal{A})$, any countably generated sub- $\sigma$-algebra $C_{0}$ of $C$ and any probability measure $Q$ on $\left(X \times Y, A \otimes C_{0}\right)$ satisfying $Q(X \times C)=P(C)$ for all $C \in C_{0}, Q$ can be factored.

(3) For any countably generated sub- $\sigma$-algebra $C_{0}$ of $C$ and any probability measure $Q$ on $\left(\mathbf{R} \times Y, B \otimes C_{0}\right)$ satisfying $Q(\mathbf{R} \times C)=P(C)$ for all $C \in C_{0}, Q$ can be factored.

Proof. $(1) \Rightarrow(2)$ Let $(X, A)$ be a measurable space, $C_{0}$ a countably generated sub- $\sigma$-algebra of $C$ and $Q$ a probability measure on $A \otimes C_{0}$ satisfying $Q(X \times C)=$ $P(C)$ for all $C \in C_{0}$. Denote by $\pi_{1}\left[\pi_{2}\right]$ the projection of $X \times Y$ onto $X[Y]$. By

Received by the editors April 15, 1985.

1980 Mathematics Subject Classification. Primary 28A12, 60A10; Secondary 28A50. 
[5, Theorem 3], the image measure $Q^{\pi_{2}}=P \mid C_{0}$ is compact. Thus, by $[\mathbf{2}, 5.3 .16]$, there exists a regular conditional probability $K$ of $\pi_{2}$ given $\pi_{1}$, i.e. $K$ is a transition probability on $X \times C_{0}$ such that

$$
Q(A \times C)=Q\left(\pi_{1}^{-1}(A) \cap \pi_{2}^{-1}(C)\right)=\int_{A} K(x, C) Q^{\pi_{1}}(d x)
$$

holds for all $A \in A$ and $C \in \mathcal{C}_{0}$. Thus $Q$ can be factored.

(2) $\Rightarrow(3)$ Trivial.

(3) $\Rightarrow(1)$ Let $f: Y \rightarrow \mathbf{R}$ be $C$-measurable. Put $Z:=f(Y)$ and denote by $\tilde{B}$ the smallest $\sigma$-algebra in $\mathbf{R}$ containing $B \cup\{Z\}$. Extend $P^{f}$ to a measure $\tilde{P}$ on $\tilde{B}$ by setting $\tilde{P}\left(\left(B_{1} \cap Z\right)+\left(B_{2}-Z\right)\right):=P^{f}\left(B_{1}\right)$ for $B_{1}, B_{2} \in B$. Next define a probability $Q_{1}$ on $\left(\mathbf{R}^{2}, B \otimes \tilde{B}\right)$ by $Q_{1}(B \times \tilde{B}):=\tilde{P}(B \cap \tilde{B}), B \in B, \tilde{B} \in \tilde{B}$. $C_{0}:=f^{-1}(B)$ is a countably generated sub- $\sigma$-algebra of $C$. For $B \in B$ and $C_{0} \in C_{0}$, say $C_{0}=f^{-1}\left(B_{0}\right)$ with $B_{0} \in B$, put $Q\left(B \times C_{0}\right):=Q_{1}\left(B \times\left(B_{0} \cap Z\right)\right)$. Then $Q$ is a well-defined probability on $B \otimes C_{0}$ satisfying

$Q\left(\mathbf{R} \times C_{0}\right)=Q_{1}\left(\mathbf{R} \times\left(B_{0} \cap Z\right)\right)=\tilde{P}\left(B_{0} \cap Z\right)=P^{f}\left(B_{0}\right)=P\left(C_{0}\right)$ for all $C_{0} \in C_{0}$.

By (3), $Q$ can be factored: $Q=\tilde{Q} \times K$, where $\tilde{Q}$ is a probability on $(\mathbf{R}, B)$ and $K$ is a transition probability on $\mathbf{R} \times C_{0}$. Then $K^{\prime}(x, \tilde{B}):=K\left(x, f^{-1}(\tilde{B} \cap Z)\right), x \in \mathbf{R}$, $\tilde{B} \in \tilde{B}$, defines a transition probability on $\mathbf{R} \times \tilde{B}$, and we obtain for $B \in B$ and $\tilde{B} \in \tilde{B}$,

$$
\begin{aligned}
\tilde{P}(B \cap \tilde{B}) & =Q_{1}(B \times \tilde{B})=Q_{1}(B \times(\tilde{B} \cap Z))=Q\left(B \times f^{-1}(\tilde{B} \cap Z)\right) \\
& =\int_{B} K\left(x, f^{-1}(\tilde{B} \cap Z)\right) \tilde{Q}(d x)=\int_{B} K^{\prime}(x, \tilde{B}) \tilde{Q}(d x),
\end{aligned}
$$

i.e.

(*) $\quad \tilde{P}(B \cap \tilde{B})=Q_{1}(B \times \tilde{B})=\int_{B} K^{\prime}(x, \tilde{B}) \tilde{Q}(d x)$ for $B \in B, \tilde{B} \in \tilde{B}$.

Setting $\tilde{B}=Z$ in $(*)$, we get $P^{f}(B)=\tilde{Q}(B)$ for $B \in B$, so $P^{f}=\tilde{Q}$. Setting $B=\tilde{B}$ in $(*)$, we obtain $P^{f}(B)=\int_{B} K^{\prime}(x, B) P^{f}(d x)$ or

$$
\int_{B}\left(1-K^{\prime}(x, B)\right) P^{f}(d x)=0 \text { for all } B \in B \text {. }
$$

Let $\mathcal{E}=\left\{B_{1}, B_{2}, \ldots\right\}$ be a countable algebra generating $B$. In view of $(* *)$, we can find, for every $n \in N$, a set $N_{n} \in B$ such that $P^{f}\left(N_{n}\right)=0$ and $1_{B_{n}}(x)$. $\left(1-K^{\prime}\left(x, B_{n}\right)\right)=0$ for $x \in \mathbf{R}-N_{n}$. Put $\tilde{N}:=\bigcup_{n \in N} N_{n}$ and $\mathcal{M}:=\{B \in B$ : $1_{B}(x) \cdot\left(1-K^{\prime}(x, B)\right)=0$ for all $\left.x \in \mathbf{R}-\tilde{N}\right\}$. Then $\mathcal{M}$ is a monotone class containing $\mathcal{E}$. This implies $\mathcal{M}=B$ and hence $1_{B}(x) \cdot\left(1-K^{\prime}(x, B)\right)=0$ for all $B \in B$ and all $x \in \mathbf{R}-\tilde{N}$. In particular, we get $K^{\prime}(x,\{x\})=1$ for all $x \in \mathbf{R}-\tilde{N}$. By construction of $K^{\prime}$, we also have $K^{\prime}(x, Z)=1$ for all $x \in \mathbf{R}$. It follows that $\mathbf{R}-\tilde{N} \subset Z$ which together with $P^{f}(\mathbf{R}-\tilde{N})=1$ implies (1).

COROLLARY 1. Let $(Y, C, P)$ be a probability space where $C$ is countably generated. Then the following three statements are equivalent:

(1) $P$ is perfect.

$\left(2^{\prime}\right)$ For any measurable space $(X, A)$ and any probability measure $Q$ on $(X \times$ $Y, A \otimes C)$ satisfying $Q(X \times C)=P(C)$ for all $C \in \mathcal{C}, Q$ can be factored. 
$\left(3^{\prime}\right)$ Every probability measure $Q$ on $(\mathbf{R} \times Y, B \otimes C)$ satisfying $Q(\mathbf{R} \times C)=P(C)$ for all $C \in C$ can be factored.

ProOF. In view of the Theorem, it suffices to prove the implications $(2) \Rightarrow\left(2^{\prime}\right)$, $\left(2^{\prime}\right) \Rightarrow\left(3^{\prime}\right)$ and $\left(3^{\prime}\right) \Rightarrow(3)$. Only the latter one is nontrivial.

Let $C_{0}$ be a countably generated sub- $\sigma$-algebra of $C$, and let $Q$ be a probability on $B \otimes C_{0}$ satisfying $Q(\mathbf{R} \times C)=P(C)$ for all $C \in C_{0}$. By means of a HahnBanach argument, combined with $[\mathbf{3}, 1(\mathrm{i})]$ (which can be applied since the marginal measure $B \in B \rightarrow Q(B \times Y)$ is Radon and hence compact), $Q$ can be extended to a probability measure $\tilde{Q}$ on $B \otimes C$ satisfying $\tilde{Q}(\mathbf{R} \times C)=P(C)$ for all $C \in \mathcal{C}$ (cf. the proof of 2.3 in $[4])$. Since, by $\left(3^{\prime}\right), \tilde{Q}$ can be factored, so can $Q$. This proves $\left(3^{\prime}\right) \Rightarrow(3)$.

COROLLARY 2. Let $(Y, C)$ be a countably generated measurable space. Then the following three statements are equivalent:

(4) Every probability measure on $C$ is perfect.

(5) For any measurable space $(X, A)$ and any probability measure $Q$ on $(X \times$ $Y, A \otimes C), Q$ can be factored.

(6) Every probability measure on $(\mathbf{R} \times Y, B \otimes C)$ can be factored.

The theorem of Blackwell and Maitra [1] is now an immediate consequence of Corollary 2 and the following proposition.

Proposition. A separable metric space $Y$ is absolutely measurable (i.e., if $\tilde{Y}$ is a metric completion of $Y$ and $\mu$ is a probability measure on $B(\tilde{Y})$, then $Y$ is $\mu$-measurable) iff every probability measure on $B(Y)$ is perfect.

Proof. According to [5, Theorem 11], the perfect probability measures on $B(Y)$ are exactly the Radon probabilities on $B(Y)$. On the other hand, any metric completion $\tilde{Y}$ of $Y$ is Polish and hence a Radon space (cf. [6, p. 122]). Thus our claim follows from Propositions 8 and 9 in [6, pp. 118-119].

REMARK. Using the methods of Pachl (cf. [4, pp. 159-161]) one can even show that every probability space $(Y, C, P)$ which satisfies condition $\left(2^{\prime}\right)$ of Corollary 1 is compact (and hence perfect). On the other hand, the complete Lebesgue measure on the unit interval, is an example of a compact probability $P$ that does not satisfy $\left(2^{\prime}\right)$.

\section{REFERENCES}

1. D. Blackwell and A. Maitra, Factorization of probability measures and absolutely measurable sets, Proc. Amer. Math. Soc. 92 (1984), 251-254.

2. P. Gänssler and W. Stute, Wahrscheinlichkeitstheorie, Springer-Verlag, Berlin, Heidelberg and New York, 1977.

3. E. Marczewski and C. Ryll-Nardzewski, Remarks on the compactness and non-direct products of measures, Fund. Math. 40 (1953), 165-170.

4. J. K. Pachl, Disintegration and compact measures, Math. Scand. 43 (1978), 157-168.

5. V. V. Sazonov, On perfect measures, Amer. Math. Soc. Transl. (2) 48 (1965), 229-254.

6. L. Schwartz, Radon measures on arbitrary topological spaces and cylindrical measures, Oxford Univ. Press, London, 1973.

Mathematisches Institut, Universität MÜnChen, Theresienstr. 39, D-8000 MÜNCHEN 2, FEDERAL REPUBLIC OF GERMANY 